\title{
Some characterizations of $T$-power based implications
}

\author{
Sebastia Massanet ${ }^{\mathrm{a}, \mathrm{b}, *}$, Jordi Recasens $^{\mathrm{c}}$, Joan Torrens ${ }^{\mathrm{a}, \mathrm{b}}$ \\ ${ }^{a}$ Soft Computing, Image Processing and Aggregation (SCOPIA) research group \\ Dept. of Mathematics and Computer Science, University of the Balearic Islands, 07122 Palma, Spain \\ ${ }^{b}$ Balearic Islands Health Research Institute (IdISBa), 07010 Palma, Spain \\ ${ }^{c}$ Secció Matemàtiques i Informàtica, ETS Arquitectura del Vallès, Universitat Politècnica de Catalunya, 08190 Sant Cugat del Vallès, Spain
}

\begin{abstract}
Recently, the so-called family of $T$-power based implications was introduced. These operators involve the use of Zadeh's quantifiers based on powers of t-norms in its definition. Due to the fact that Zadeh's quantifiers constitute the usual method to modify fuzzy propositions, this family of fuzzy implication functions satisfies an important property in approximate reasoning such as the invariance of the truth value of the fuzzy conditional when both the antecedent and the consequent are modified using the same quantifier. In this paper, an in-depth analysis of this property is performed by characterizing all binary functions satisfying it. From this general result, a fully characterization of the family of $T$-power based implications is presented. Furthermore, a second characterization is also proved in which surprisingly the invariance property is not explicitly used.
\end{abstract}

Key words: Fuzzy implication function, $T$-power based implications, Invariance property, continuous t-norm, powers of t-norms.

\section{Introduction}

The study of fuzzy implication functions has experienced a considerable growth in last decades because of their great quantity of applications that are not limited to approximate reasoning and fuzzy control. Quite the opposite they extend to many other fields like image processing, fuzzy relational equations, fuzzy DI-subsethood measures, computing with words, data mining and rough sets, and so on (see [3, 5, 6] and the references within). For this reason, it is important to have a wide range of possibilities as well as many different models of fuzzy implication functions in order to find the most adequate operator for each context [28]. All of this has led to a systematic analysis of the theoretical properties of fuzzy implication functions, including the introduction of several construction methods, the study of new families and the research of their possible axiomatic characterizations.

There are lots of different models to construct fuzzy implication functions that can be mainly classified in three main different strategies:

1. Implications obtained combining aggregation functions and other logical connectives. This includes material implications based on the classical model $p \rightarrow q \equiv \neg p \vee q$, residual implications based on the residuation property in lattices, $Q L$-implications based on quantum logic and also $D$-implications. All these models are usually constructed from t-norms, t-conorms and fuzzy negations, but recently they have been also constructed from conjunctive and disjunctive uninorms, as well as from more general aggregation functions (see [6] and the references therein and [25]). The most general study in this line leading to a bijection between implications and conjunctors (or disjunctors) can be found in [25]. Other approaches generating fuzzy implication functions from copulas can be found in [10,11] and from semicopulas in [4].

\footnotetext{
*Corresponding author. Phone: +34971259915

Email addresses: s.massanet@uib.es (Sebastia Massanet), j.recasens@upc.edu (Jordi Recasens), jts224@uib.es (Joan Torrens)
} 
2. Implications generated from unary functions. This class of implications is based on the use of additive generating functions leading to well-known families such as Yager's $f$ - and $g$-generated implications [32] or $h$ and $(h, e)$-implications [18]. Again some generalizations have been also proposed (see [12]).

3. Implications constructed from other implications. This strategy includes well-knowm methods like the $\varphi$ conjugate, the convex linear combinations, and the upper, lower and medium contrapositivizations (see [5]). In this line, again many new possibilities have appeared as for instance in [2, 20, 21, 27, 29, 30].

Each one of the above mentioned families satisfy particular properties that make them adequate for one or either purpose. For this reason, the investigation of possible characterizations of each of these families through some algebraic properties has been a common objective by many researchers (see for instance $[1,5,19]$ ).

Recently, a new important property was studied in [23] and called there invariance with respect to powers of a continuous t-norm $T$. Although this property is not usually required on fuzzy implication functions, it is closely related to approximate reasoning. It was suggested through the well known example on tomatoes in [24] based on the argument that the value of a conditional should be invariant with respect to some linguistic modifiers in both, the antecedent and the consequent. In [23] the property was revisited by modelling these linguistic modifiers through powers of continuous t-norms (see [31] and also [7] and [15] for powers of other aggregation functions).

Invariance with respect to powers of a continuous t-norm $T$ is not satisfied by the usual families of fuzzy implication functions [23]. Thus, in that paper the authors introduce a new family of fuzzy implication functions, called $T$-power based implications, that satisfies such invariance property. Following this line, in this paper we want to study $T$-power based implications, investigate new properties and give two different axiomatic characterizations of this family. The first one precisely based in the invariance property with respect to $T$-powers and the other one based on different properties.

The structure of the paper is as follows. In Section 2, the basic concepts and results related to powers of t-norms and fuzzy implication functions will be collected, as well as the definition of the recently introduced family of fuzzy implication functions based on powers of t-norms and its structure. In Section 3 an in-depth study on the invariance property with respect to powers of t-norms is performed from which a general characterization of all binary functions satisfying it is provided, leading then to the mentioned characterization of $T$-power based implications based on such invariance property. Section 4 deals with an alternative characterization based on other properties of $T$-power based implications. Finally, the paper ends with a section devoted to the conclusions.

\section{Preliminaries}

We will suppose the reader is familiar with basic results on t-norms, t-conorms, fuzzy negations and fuzzy implication functions, see $[14,31]$ for more details on t-norms and see $[3,5,6,9]$ for more details on fuzzy implication functions. In this section we will recall only some concepts about powers with respect to continuous t-norms and also about fuzzy implication functions to make the paper as self-contained as possible.

\subsection{Powers with respect to continuous t-norms}

We will suppose that all t-norms $T$ used in this section and throughout the paper are continuous. For more details about the results included in this section see [31] where powers with respect to continuous t-norms are fully studied.

From the associativity of any t-norm $T$, integer powers with respect to $T$ can be defined in the usual way, that is,

$$
x_{T}^{(n)}=T(\overbrace{x, x, \ldots, x}^{n \text { times }}) \quad \text { for all } x \in[0,1], n \in \mathbb{Z}^{+} \text {and } n \geq 2,
$$

with the conventions $x_{T}^{(1)}=x$ and $x_{T}^{(0)}=1$ for all $x \in[0,1]$.

Similarly, $n$-th roots and rational powers of an element $x \in[0,1]$ with respect to a t-norm $T$ are defined as

$$
x_{T}^{\left(\frac{1}{n}\right)}=\sup \left\{z \in[0,1] \mid z^{(n)} \leq x\right\}, \quad x_{T}^{\left(\frac{m}{n}\right)}=\left(x_{T}^{\left(\frac{1}{n}\right)}\right)^{(m)}
$$

for all $m, n \in \mathbb{Z}^{+}$. 
Lemma 1 ([31]). Consider $k, m, n \in \mathbb{Z}^{+}$and let $T$ be a continuous $t$-norm. Then $x_{T}^{\left(\frac{k m}{k n}\right)}=x_{T}^{\left(\frac{m}{n}\right)}$ for all $x \in[0,1]$.

From the continuity of $T$, rational powers with respect to $T$ can be extended to irrational powers through the following definition.

Definition 1 ([31]). Let $T$ be a continuous t-norm and $r \in \mathbb{R}^{+}$a positive real number. Consider $\left\{a_{n}\right\}_{n \in \mathbb{Z}^{+}}$a sequence of rational numbers such that $\lim _{n \rightarrow \infty} a_{n}=r$. For all $x \in[0,1]$, the power $x_{T}^{(r)}$ is defined as

$$
x_{T}^{(r)}=\lim _{n \rightarrow \infty} x_{T}^{\left(a_{n}\right)}
$$

We can extend the definition to $r=+\infty$ in the same way and then

$$
x_{T}^{(+\infty)}=\lim _{n \rightarrow \infty} x_{T}^{\left(a_{n}\right)}
$$

where $\left\{a_{n}\right\}_{n \in \mathbb{Z}^{+}}$is a sequence of rational numbers such that $\lim _{n \rightarrow \infty} a_{n}=+\infty$.

The continuity of $T$ ensures both, the existence of the limit and the independence of the considered sequence $\left\{a_{n}\right\}_{n \in \mathbb{Z}^{+}}$. It is immediate to check that $0 \leq x_{T}^{(r)} \leq 1$ and $x_{T}^{(r)} \leq y_{T}^{(r)}$ whenever $x \leq y$ for all $x, y \in[0,1]$ and $r \in[0,+\infty]$.

When the selected t-norm is Archimedean, the expressions of these powers only depend on the additive generator of the t-norm. Recall that additive generators of continuous Archimedean t-norms are continuous, strictly decreasing functions $f:[0,1] \rightarrow[0,+\infty]$ with $f(1)=0$.

Proposition 2 ([31]). Let $T$ be a continuous Archimedean t-norm with additive generator $t$. Then

$$
x_{T}^{(r)}=t^{-1}(\min \{t(0), r t(x)\}) \text { for all } x \in[0,1] \text { and } r \in[0,+\infty]
$$

with the convention that $0 \cdot \infty=0$.

Example 1. In the cases of the three basic continuous t-norms we have that for all $x \in[0,1]$ :

- When $T(x, y)=T_{\mathbf{L}}(x, y)=\max \{x+y-1,0\}$ is the Łukasiewicz t-norm, then $x_{T_{\mathbf{L}}}^{(r)}=\max \{0,1-r+r x\}$.

- When $T(x, y)=T_{\mathbf{P}}(x, y)=x y$ is the Product t-norm, then $x_{T_{\mathbf{P}}}^{(r)}=x^{r}$.

- When $T(x, y)=T_{\mathbf{M}}(x, y)=\min \{x, y\}$ is the Minimum t-norm, then $x_{T_{\mathbf{M}}}^{(r)}= \begin{cases}x & \text { if } r>0 \\ 1 & \text { if } r=0 \text {. }\end{cases}$

\subsection{Fuzzy implication functions}

First, we recall the definition of a fuzzy implication function.

Definition 2 ([5, 9]). A binary operation $I:[0,1]^{2} \rightarrow[0,1]$ is said to be a fuzzy implication function if it satisfies:

(I1) $I(x, z) \geq I(y, z)$ when $x \leq y$, for all $z \in[0,1]$.

(I2) $I(x, y) \leq I(x, z)$ when $y \leq z$, for all $x \in[0,1]$.

(I3) $I(0,0)=I(1,1)=1$ and $I(1,0)=0$.

It follows from the definition that $I(0, x)=1$ and $I(x, 1)=1$ for all $x \in[0,1]$ whereas the symmetrical values $I(x, 0)$ and $I(1, x)$ are not derived from the definition. Fuzzy implication functions can satisfy additional properties usually coming from tautologies in crisp logic. Let us recall here some of the most usual ones.

Definition $3([5,9])$. Let $I$ be a fuzzy implication function. 
- The function $N_{I}$ defined by $N_{I}(x)=I(x, 0)$ for all $x \in[0,1]$, is called the natural negation of $I$ and it is always a fuzzy negation.

- $I$ can additionally satisfy the following properties:

1. Exchange Principle:

$$
I(x, I(y, z))=I(y, I(x, z)), \quad \text { for all } x, y, z \in[0,1] .
$$

2. Law of importation with respect to a t-norm $T$ :

$$
I(T(x, y), z)=I(x, I(y, z)), \quad \text { for all } \quad x, y, z \in[0,1] .
$$

3. Left-neutrality principle:

$$
I(1, y)=y \quad \text { for all } \quad y \in[0,1]
$$

4. Ordering Property:

$$
x \leq y \quad \Longleftrightarrow \quad I(x, y)=1 \quad \text { for all } \quad x, y \in[0,1]
$$

5. Identity Principle:

$$
I(x, x)=1 \text { for all } x \in[0,1] .
$$

6. The contrapositive symmetry with respect to a fuzzy negation $N$,

$$
I(x, y)=I(N(y), N(x)), \quad \text { for all } x, y \in[0,1] .
$$

7. The T-transitivity with respect to a t-norm $T$,

$$
T(I(x, y), I(y, z)) \leq I(x, z) \quad \text { for all } \quad x, y, z \in[0,1]
$$

Recently, it was introduced in [23] a new family of fuzzy implication functions based on the idea of relaxing or intensifying the antecedent of a fuzzy IF-THEN rule through the use of the Zadeh's linguistic powering modifiers [13]. Specifically, the truth value of a conditional $x \rightarrow y$ was defined as the highest possible power of $y$ (with respect to a continuous t-norm $T$ ) such that $y$ up to this power becomes greater than or equal to $x$. For instance, $(0.8 \rightarrow 0.64)=\frac{1}{2}$ because of, using the product t-norm we have $0.64^{\frac{1}{2}}=0.8$.

From this previous idea the formal definition was given in [23] as follows.

Definition 4 ([23]). A binary operator $I:[0,1]^{2} \rightarrow[0,1]$ is said to be a $T$-power based implication if there exists a continuous t-norm $T$ such that

$$
I(x, y)=\sup \left\{r \in[0,1] \mid y_{T}^{(r)} \geq x\right\} \quad \text { for all } x, y \in[0,1] .
$$

If $I$ is a $T$-power based implication, then it will be denoted by $I^{T}$.

Based on the previous definition one can easily prove that $T$-power based implications, $I^{T}$, are in fact fuzzy implication functions in the sense of Definition 2 for any continuous t-norm $T$. Moreover, the general expression of the $T$-power based implications was proved in [23] depending on the structure of the continuous t-norm $T$ used in the process, as follows.

Proposition 3 ([23]). Let $T$ be a continuous t-norm and $I^{T}$ its power based implication.

- If $T=T_{\mathrm{M}}$ is the minimum t-norm, then $I^{T_{\mathrm{M}}}$ agrees with the Rescher implication, that is:

$$
I^{T_{\mathbf{M}}}(x, y)=I_{\mathbf{R S}}(x, y)= \begin{cases}1 & \text { if } x \leq y \\ 0 & \text { if } x>y\end{cases}
$$


- If $T$ is an Archimedean t-norm with additive generator $t$, then

$$
I^{T}(x, y)= \begin{cases}1 & \text { if } x \leq y \\ \frac{t(x)}{t(y)} & \text { if } x>y\end{cases}
$$

with the convention that $\frac{a}{+\infty}=0$ for all $a \in[0,+\infty[$.

- If $T$ is an ordinal sum $t$-norm of the form $T=\left(\left\langle a_{j}, b_{j}, T_{j}\right\rangle\right)_{j \in J}$, where $T_{j}$ is an Archimedean t-norm with additive generator $t_{j}$ for all $j \in J$, then

$$
I^{T}(x, y)= \begin{cases}1 & \text { if } x \leq y, \\ \frac{t_{j}\left(\frac{x-a_{j}}{b_{j}-a_{j}}\right)}{t_{j}\left(\frac{y-a_{j}}{b_{j}-a_{j}}\right)} & \text { if } x, y \in\left[a_{j}, b_{j}\right] \quad \text { and } x>y, \\ 0 & \text { otherwise, }\end{cases}
$$

with the convention that $\frac{a}{+\infty}=0$ for all $a \in[0,+\infty[$.

From this structure, it is easily proved (see [23]) that fuzzy implication functions $I^{T}$ always satisfy (IP) and (OP) for any continuous t-norm $T$. On the contrary, they never satisfy either $(\mathbf{N P}),(\mathbf{E P})$ or $\left(\mathbf{L} \mathbf{I}_{\mathbf{T}}\right)$ for any t-norm. On the other hand, there are other properties like $(\mathbf{C P}(\mathbf{N}))$ or $T$-transitivity that implications $I^{T}$ satisfy, but only in some cases, see again [23] for more details.

\section{Characterizing $T$-power based implications through invariance with respect to powers of continuous t- norms}

As we have already commented in the introduction, the most important property of $T$-power based implications, $I^{T}$, is the invariance with respect to linguistic modifiers modelled through powers of t-norms. Specifically, we have the following definition.

Definition 5. Let $I:[0,1]^{2} \rightarrow[0,1]$ be a binary function. It is said that $I$ is invariant with respect to $T$-powers, or simply that it is $T$-power invariant when

$$
I(x, y)=I\left(x_{T}^{(r)}, y_{T}^{(r)}\right)
$$

holds for all real number $r>0$ and for all $x, y \in[0,1]$ such that $x_{T}^{(r)}, y_{T}^{(r)} \neq 0,1$.

Remark 1. Note that when $T$ is the minimum t-norm the property $\left(\mathbf{P I}_{\mathbf{T}}\right)$ is trivially satisfied for any fuzzy implication function $I$ because in this case we have $x_{T}^{(r)}=x$ for all $r>0$. That is, any fuzzy implication function $I$ is min-power invariant. On the other hand, the Rescher implication $I_{\mathbf{R S}}$ which is the min-power based implication (see Proposition 3), clearly satisfies that it is $T$-power invariant with respect to any continuous t-norm $T$.

With this definition we have the following result for $I^{T}$.

Proposition 4 ([23]). Let $T$ be a continuous t-norm and $I^{T}$ its power based implication. Then $I^{T}$ is $T$-power invariant.

As it is stated in [23], the fulfillment of this property ensures that the following fuzzy propositions from the classical example given in [24]:

If the tomato is red, then it is ripe.

If the tomato is very red, then it is very ripe.

If the tomato is little red, then it is little ripe. 
have the same truth value whenever the linguistic modifiers "very" and "little" are modeled using powers of continuous t-norms.

Taking into account that the most usual fuzzy implication functions do not satisfy property $\left(\mathbf{P I}_{\mathbf{T}}\right)$ (see [23]), the previous result already shows a close relation between the $T$-power invariance property and the class of $T$-power based implications. Moreover, we will see in this paper that this relation is even closer than the result stated in Proposition 4. In fact we will prove that the $T$-power invariance property characterizes itself, jointly with (IP), the class of $T$-power based implications up to compositions with increasing mappings.

Since in this paper we deal with continuous t-norms and taking into account Remark 1, we will divide our reasoning in two sections, one devoted to the Archimedean case and the other devoted to the continuous ordinal sum case.

\subsection{The case when $T$ is Archimedean}

First we will deal with continuous Archimedean t-norms. Although the final result is the same for both subcases: the strict and the nilpotent case, the corresponding proofs present some differences and so we need to study both cases separately. However, some initial results can be stated jointly.

First of all recall that additive generators of Archimedean t-norms are unique up to a positive multiplicative constant. Thus, in the case of continuous Archimedean t-norms, when $T$ is strict it is $t(0)=+\infty$ for any additive generator $t$ and, when $T$ is nilpotent, it can be considered an additive generator $t$ with any finite value of $t(0)<+\infty$ (in particular, the one with $t(0)=1$ is usually called normalized additive generator)..

The following lemma will be useful in the forthcoming characterization results.

Lemma 5. Let $T$ be an Archimedean $t$-norm and $t$ an additive generator of $T$ with $t(0)>1$. Then

$$
x=\left(t^{-1}(1)\right)_{T}^{(t(x))} \quad \text { for all } x \in[0,1] .
$$

Proof. Note that with the assumption that $t(0)>1$ there exists $t^{-1}(1) \in[0,1]$. Thus, following Proposition 2, we have that

$$
\left(t^{-1}(1)\right)_{T}^{(t(x))}=t^{-1}\left(\min \left(t(0), t(x) \cdot t\left(t^{-1}(1)\right)\right)\right)=t^{-1}(\min (t(0), t(x)))=x .
$$

for all $x \in[0,1]$.

Lemma 6. Let $T$ be an Archimedean t-norm and let $I:[0,1]^{2} \rightarrow[0,1]$ be a function invariant with respect to $T$-powers. Then $I(x, x)$ is constant for all $x \in] 0,1[$.

Proof. This is simply due to the fact that when $T$ is Archimedean the $T$-powers of any $x \in] 0,1[$ take all values in the open unit interval. Thus, fixed some $a \in] 0,1[$, for all $x \in] 0,1\left[\right.$ there exists $r>0$ such that $x=a_{T}^{(r)}$ and then by $T$-power invariance

$$
I(x, x)=I\left(a_{T}^{(r)}, a_{T}^{(r)}\right)=I(a, a) .
$$

The previous result shows that in the Archimedean case the $T$-power invariance property is closely related with the property (IP) for fuzzy implication functions. Effectively, if $I$ is a $T$-power invariant fuzzy implication function and there exists some $x \in] 0,1[$ such that $I(x, x)=1$, then $I$ satisfies $(\mathbf{I P})$.

\subsubsection{Strict Archimedean t-norms}

Let us now deal with the strict case. The following proposition shows how strong is the $T$-power invariance property, specially when $T$ is strict.

Proposition 7. Let $T$ be a continuous Archimedean strict t-norm and $t$ an additive generator of $T$. A mapping $I:[0,1]^{2} \rightarrow[0,1]$ is invariant with respect to $T$-powers if and only if there exists a mapping $\left.\varphi:\right] 0,+\infty[\rightarrow[0,1]$ such that $I$ is given by

$$
\left.I(x, y)=\varphi\left(\frac{t(x)}{t(y)}\right)_{6} \text { for all } x, y \in\right] 0,1[
$$


Proof. Let us first suppose that $I$ is given by Equation (1). Since $T$ is strict, $t(0)=+\infty$ and then applying Proposition 2 we obtain:

$$
I\left(x_{T}^{(r)}, y_{T}^{(r)}\right)=\varphi\left(\frac{t\left(x_{T}^{(r)}\right)}{t\left(y_{T}^{(r)}\right)}\right)=\varphi\left(\frac{t\left(t^{-1}(r t(x))\right)}{t\left(t^{-1}(r t(y))\right)}\right)=\varphi\left(\frac{t(x)}{t(y)}\right)=I(x, y)
$$

for all $x, y \in] 0,1[$ and for all $r>0$.

Conversely, if $I$ is $T$-power invariant, again by Proposition 2 and Lemma 6 we have

$$
\begin{aligned}
I(x, y) & =I\left(x,\left(t^{-1}(1)\right)_{T}^{(t(y))}\right)=I\left(x_{T}^{\left(\frac{1}{t(y)}\right)(t(y))},\left(t^{-1}(1)\right)_{T}^{(t(y))}\right) \\
& =I\left(x_{T}^{\left(\frac{1}{t(y)}\right)}, t^{-1}(1)\right)=I\left(t^{-1}\left(\frac{1}{t(y)} \cdot t(x)\right), t^{-1}(1)\right)
\end{aligned}
$$

for all $x, y \in] 0,1[$. Thus, if we consider the function $\varphi:] 0,+\infty\left[\rightarrow[0,1]\right.$ given by $\varphi(z)=I\left(t^{-1}(z), t^{-1}(1)\right)$, by the previous equality we obtain

$$
\left.I(x, y)=\varphi\left(\frac{t(x)}{t(y)}\right) \quad \text { for all } \quad x, y \in\right] 0,1[
$$

and the result follows.

Now, seeking for fuzzy implication functions $I$, we must additionally require that $I$ is decreasing in its first coordinate and increasing in its second coordinate and also that $I(0,0)=I(1,1)=1$ and $I(1,0)=0$. These conditions translated to function $\varphi$ lead to the following theorem.

Theorem 8. Let $T$ be a strict t-norm and $t$ an additive generator of $T$. A mapping $I:[0,1]^{2} \rightarrow[0,1]$ is a fuzzy implication function invariant with respect to T-powers if and only if there exists an increasing mapping $\varphi:[0, \infty] \rightarrow$ $[0,1]$ with $\varphi(0)=0, \varphi(+\infty)=1$ and such that $I$ is given by

$$
I(x, y)=\varphi\left(\frac{t(x)}{t(y)}\right) \quad \text { for all }(x, y) \in[0,1]^{2} \backslash\{(x, 0),(1, y) \mid 0<x, y<1\},
$$

with the convention that $\frac{0}{0}=\frac{+\infty}{+\infty}=+\infty$, and such that the remaining values $I(x, 0)$ and $I(1, y)$ preserve the monotonicity conditions.

Proof. By Proposition 7 we already know the equivalence with the existence of a mapping $\varphi:] 0,+\infty[\rightarrow[0,1]$ satisfying Equation (2) for all $x, y \in] 0,1\left[\right.$. Note that when $x, y$ are different from 0,1 we have $\frac{t(x)}{t(y)} \neq 0,+\infty$ and so the values $\varphi(0)$ and $\varphi(+\infty)$ can be anyone. However, we will have $I(1,0)=0$ if and only if Equation (2) also holds for $(1,0)$, by taking $\varphi(0)=0$ since

$$
0=I(1,0)=\varphi\left(\frac{t(1)}{t(0)}\right)=\varphi\left(\frac{0}{+\infty}\right)=\varphi(0)
$$

Similarly, we will have $I(0, y)=1$ for all $y<1$ and $I(x, 1)=1$ for all $x<1$ if and only if Equation (2) also holds for these values $(0, y)$ and $(x, 1)$, by taking $\varphi(+\infty)=1$ because

$$
1=I(0, y)=\varphi\left(\frac{t(0)}{t(y)}\right)=\varphi(+\infty) \quad \text { and } \quad 1=I(x, 1)=\varphi\left(\frac{t(x)}{t(1)}\right)=\varphi(+\infty)
$$

Moreover, we will have also $I(0,0)=I(1,1)=1$ if and only if we impose Equation (2) to the values $(0,0)$ and $(1,1)$ just by assuming the convention $\frac{0}{0}=\frac{+\infty}{+\infty}=+\infty$.

Finally, from Equation (2) and the previous reasoning, it is clear that function $I$ satisfies monotonicities (I1), (I2) in Definition 2 if and only if $\varphi$ is an increasing mapping and the remaining values $I(x, 0)$ and $I(1, y)$ with $0<x, y<1$ preserve these monotonicity conditions. 


\section{Remark 2. Some remarks about the previous result are needed:}

i) The convention $\frac{0}{0}=\frac{+\infty}{+\infty}=+\infty$ can be substituted by $\frac{0}{0}=\frac{+\infty}{+\infty}=a$ where $a$ is any value satisfying that $\varphi(a)=1$.

ii) Note that examples of fuzzy implication functions $I:[0,1]^{2} \rightarrow[0,1]$ satisfying the properties stated in the theorem above are available just by taking the values $I(x, 0)=I(1, y)=0$ for all $0<x, y<1$. It is straightforward to check that with these values the monotonicity properties are always preserved.

Note that, although $I(x, x)$ is constant for all $x \in] 0,1[$ for this kind of implications, $($ IP $)$ is not guaranteed, as the following example shows.

Example 2. Take some value $a \in] 0,1[$ and consider the fuzzy implication function given by:

$$
I_{1}(x, y)= \begin{cases}1 & \text { if } x=0 \text { or } y=1 \\ a & \text { if } x \neq 0,1 \text { and } y \neq 0,1 \\ 0 & \text { otherwise }\end{cases}
$$

It is clear that $I_{1}$ is a $T$-power invariant fuzzy implication function for any Archimedean t-norm $T$ (strict or nilpotent), but $I_{1}$ does not satisfy (IP). Note that, for any strict t-norm $T, I_{1}$ is obtained through Equation (2) by taking the function $\varphi:[0,+\infty] \rightarrow[0,1]$ given by

$$
\varphi(x)= \begin{cases}0 & \text { if } x=0 \\ a & \text { if } x \neq 0,+\infty \\ 1 & \text { if } x=+\infty\end{cases}
$$

and the corresponding values $I(x, 0)=I(1, y)=0$. However, note that the fuzzy implication function $I_{2}$ given by

$$
I_{2}(x, y)= \begin{cases}1 & \text { if } x=0 \text { or } y=1 \\ 0 & \text { if }(x, y)=(1,0) \\ a & \text { otherwise }\end{cases}
$$

is also obtained through Equation (2) by taking the same function $\varphi:[0,+\infty] \rightarrow[0,1]$, but considering the remaining values $I(x, 0)=I(1, y)=a$, that also preserve the monotonicity conditions.

In fact, when $T$ is strict, $T$-power invariant fuzzy implication functions satisfy (IP) if and only if the corresponding function $\varphi$ satisfies $\varphi(1)=1$ as it is stated in the following result.

Theorem 9. Let $T$ be a strict $t$-norm and $t$ an additive generator of $T$. A mapping $I:[0,1]^{2} \rightarrow[0,1]$ is a fuzzy implication function invariant with respect to $T$-powers and satisfying $(\mathbf{I P})$ if and only if there exists an increasing mapping $\varphi:[0,+\infty] \rightarrow[0,1]$ with $\varphi(0)=0, \varphi(1)=1$ and such that $I$ is given by

$$
I(x, y)=\varphi\left(\frac{t(x)}{t(y)}\right) \quad \text { for all }(x, y) \in[0,1]^{2} \backslash\{(x, 0),(1, y) \mid 0<x, y<1\},
$$

with the convention that $\frac{0}{0}=\frac{+\infty}{+\infty}=1$, and such that the remaining values $I(x, 0)$ and $I(1, y)$ preserve the monotonicity conditions.

Moreover, in this case I satisfies (OP) if and only if $\varphi(x)<1$ for all $x<1$.

Proof. This is clear from Theorem 8 since for all $x \in] 0,1[$ we have that $I(x, x)=1$ if and only if $\varphi(1)=1$ and in this case, by Remark 2-i), the convention becomes $\frac{0}{0}=\frac{+\infty}{+\infty}=\varphi^{-1}(1)=1$.

On the other hand, by increasingness we have $I(x, y)=1$ whenever $x \leq y$, whereas for $x>y$ it is $I(x, y)<1$ if and only if $\varphi(x)<1$ for all $x<1$. Thus, $I$ satisfies $(\mathbf{O P})$ if and only if $\varphi(x)<1$ for all $x<1$. 
In the result above, since $\varphi:[0, \infty] \rightarrow[0,1]$ is an increasing mapping and $\varphi(1)=1$, we have $\varphi(z)=1$ for all $z \geq 1$, which implies that $I(x, y)=1$ when $x \leq y$. Thus, the previous theorem can be written in the following alternative form.

Theorem 10. Let $T$ be a strict t-norm and $t$ an additive generator of $T$. A mapping $I:[0,1]^{2} \rightarrow[0,1]$ is a fuzzy implication function invariant with respect to $T$-powers and satisfying $(\mathbf{I P})$ if and only if there exists an increasing mapping $\varphi:[0,1] \rightarrow[0,1]$ with $\varphi(0)=0$ such that $I$ is given by

$$
I(x, y)= \begin{cases}1 & \text { if } x \leq y, \\ \varphi\left(\frac{t(x)}{t(y)}\right) & \text { if } 1>x>y>0 \text { or }(x, y)=(1,0),\end{cases}
$$

with the convention that $\frac{0}{0}=\frac{+\infty}{+\infty}=1$, and such that the remaining values $I(x, 0)$ and $I(1, y)$ preserve the monotonicity conditions.

Remark 3. Note that in the last theorem condition $\varphi(1)=1$ needs not to be assumed because in Equation (4) $\varphi$ is only applied to values less than 1 .

\subsubsection{Nilpotent Archimedean t-norms}

Let us now deal with the nilpotent case that presents some differences. First of all, an analogous result as in Proposition 7 is not available in this case but we can ensure the following partial result.

Proposition 11. Let $T$ be a nilpotent $t$-norm and $t$ an additive generator of $T$. If a mapping $I:[0,1]^{2} \rightarrow[0,1]$ is invariant with respect to $T$-powers, then there exists a mapping $\varphi:] 0,+\infty[\rightarrow[0,1]$ such that I is given by

$$
\left.I(x, y)=\varphi\left(\frac{t(x)}{t(y)}\right) \quad \text { for all } x, y \in\right] 0,1\left[\text { such that } \frac{t(x)}{t(y)}<1 .\right.
$$

PROOF. Let us first consider an additive generator $t$ with $t(0)>1$. If $T$ is nilpotent, we have that $x_{T}^{(r)} \neq 0$ if and only if $r t(x)<t(0)$ by Proposition 2. Thus, analogously as in the proof of Proposition 7 we obtain

$$
\begin{aligned}
I(x, y) & =I\left(x,\left(t^{-1}(1)\right)_{T}^{(t(y))}\right)=I\left(x_{T}^{\left(\frac{1}{t(y)}\right)(t(y))},\left(t^{-1}(1)\right)_{T}^{(t(y))}\right) \\
& =I\left(x_{T}^{\left(\frac{1}{t(y)}\right)}, t^{-1}(1)\right)=I\left(t^{-1}\left(\min \left(t(0), \frac{1}{t(y)} \cdot t(x)\right)\right), t^{-1}(1)\right),
\end{aligned}
$$

for all $x, y \in] 0,1\left[\right.$ such that $\min \left(t(0), \frac{1}{t(y)} \cdot t(x)\right) \neq t(0)$, that is, whenever $\frac{t(x)}{t(y)}<t(0)$. Thus, if we consider the function $\varphi:[0,+\infty] \rightarrow[0,1]$ given by $\varphi(z)=I\left(t^{-1}(z), t^{-1}(1)\right)$, the result is true for this generator $t$. However, since any other generator differs from $t$ only by a positive multiplicative constant, it is clear that the values $\frac{t(x)}{t(y)}$ coincide for all possible generators, not necessarily those such that $t(0)>1$, and so the result follows.

However, this partial result is enough to prove an analogous result to Theorem 10 for nilpotent t-norms as follows.

Theorem 12. Let $T$ be a nilpotent $t$-norm and $t$ an additive generator of $T$. A mapping $I:[0,1]^{2} \rightarrow[0,1]$ is a fuzzy implication function invariant with respect to $T$-powers and satisfying $(\mathbf{I P})$ if and only if there exists an increasing mapping $\varphi:[0,1] \rightarrow[0,1]$ with $\varphi(0)=0$ such that $I$ is given by

$$
I(x, y)= \begin{cases}1 & \text { if } x \leq y, \\ \varphi\left(\frac{t(x)}{t(y)}\right) & \text { if } 1>x>y>0 \text { or }(x, y)=(1,0),\end{cases}
$$

with the convention that $\frac{0}{0}=\frac{+\infty}{+\infty}=1$, and such that the remaining values $I(x, 0)$ and $I(1, y)$ preserve the monotonicity conditions. 
Proof. On the one hand, if $I$ is given by Equation (6) with the convention that $\frac{0}{0}=\frac{+\infty}{+\infty}=1$ and with values $I(x, 0)$ and $I(1, y)$ preserving the monotonicity conditions, then $I$ is clearly a fuzzy implication function satisfying (IP). Moreover, take $x, y$ such that $x_{T}^{(r)}, y_{T}^{(r)} \neq 0,1$ and let us prove that $I$ is $T$-power invariant in two cases:

- If $x \leq y$, then $x_{T}^{(r)} \leq y_{T}^{(r)}$ for all $r>0$ and consequently $I(x, y)=I\left(x_{T}^{(r)}, y_{T}^{(r)}\right)=1$.

- If $x>y$, since $1>x_{T}^{(r)}>y_{T}^{(r)}>0$ we have

$$
I\left(x_{T}^{(r)}, y_{T}^{(r)}\right)=\varphi\left(\frac{t\left(x_{T}^{(r)}\right)}{t\left(y_{T}^{(r)}\right)}\right)=\varphi\left(\frac{r t(x)}{r t(y)}\right)=I(x, y) .
$$

Conversely, if $I$ is a fuzzy implication function satisfying (IP), then clearly $I(x, y)=1$ for all $x \leq y$. Moreover, if $I$ is $T$-power invariant, then by Proposition 11 there is an increasing mapping $\varphi:] 0,+\infty] \rightarrow[0,1]$ such that $I$ is given by Equation 5. Let us name also by $\varphi$ its restriction to the interval $[0,1]$ and considering $\varphi(0)=0$. Note that when $1>x>y>0$ we have $0<\frac{t(x)}{t(y)}<1<t(0)$ and then Equation (6) follows.

\subsubsection{Analysis of the results}

Theorems 10 and 12 characterize a more general class of fuzzy implication functions than the class of $T$-power based implication functions as we can see in the following example.

Example 3. Conditions on function $\varphi:[0,1] \rightarrow[0,1]$ of Theorems 10 and 12 are quite simple and consequently there is a plethora of functions $\varphi$ satisfying them. For instance,

i) If $\varphi$ is the identity map, then we recover the power based implication $I^{T}$ with respect to $T$, but also many others varying for instance the values on $I(x, 0)$. In particular, if $T$ is a nilpotent Archimedean t-norm with a normalized additive generator $t$, we obtain also the following fuzzy implication functions:

$$
I(x, y)= \begin{cases}k t(x) & \text { if } y=0 \text { and } x \in] 0,1[, \\ I^{T}(x, y) & \text { otherwise, }\end{cases}
$$

when $k$ is any constant with $k<1$.

ii) If $\varphi(x)=\frac{\log (x+1)}{\log 2}$ then we obtain, among others, fuzzy implication functions like

$$
I(x, y)= \begin{cases}1 & \text { if } x \leq y \\ \frac{\log \left(\frac{t(x)+t(y)}{t(y)}\right)}{\log 2} & \text { if } x>y\end{cases}
$$

which is different from any $T$-power based implication.

iii) If $\varphi(x)=0$ for all $x<1$ and $\varphi(1)=1$ (note that from Remark 3 even the 0-mapping could be considered), then we obtain the Rescher implication (which agrees with the min-power based implication), that is,

$$
I(x, y)=I_{\mathbf{R S}}(x, y)= \begin{cases}1 & \text { if } x \leq y \\ 0 & \text { if } x>y\end{cases}
$$

iv) If $\varphi(0)=0$ and $\varphi(x)=1$ for $x>0$, then we obtain the fuzzy implication function

$$
I(x, y)= \begin{cases}0 & \text { if }(x=1, y<1) \text { or }(x>0, y=0) \\ 1 & \text { otherwise }\end{cases}
$$

The structure of the fuzzy implication functions characterized in Theorems 10 and 12 when the continuous t-norm is Archimedean can be viewed in Figure 1. 
Figure 1: Structure of the fuzzy implication functions satisfying (IP) and the invariance with respect to $T$-powers when $T$ is a continuous Archimedean t-norm with additive generator $t$, where the values $I(x, 0)$ and $I(1, y)$ when $0<x, y<1$ are anyones preserving monotonicities. Moreover, $\varphi:[0,1] \rightarrow[0,1]$ is an increasing mapping such that $\varphi(0)=0$.

\subsection{The general ordinal sum case}

In this section we want to give a similar characterization theorem in the general continuous case, that is, when $T$ is an ordinal sum of the form $T=\left(\left\langle a_{j}, b_{j}, T_{j}\right\rangle\right)_{j \in J}$, where $T_{j}$ is a continuous Archimedean t-norm with additive generator $t_{j}$ for all $j \in J$, see [14] for the notation on ordinal sum t-norms. In this case we will do it jointly with the (IP) condition. Thus, we can state the following result.

Theorem 13. Let $T$ be a continuous ordinal sum $t$-norm of the form $T=\left(\left\langle a_{j}, b_{j}, T_{j}\right\rangle\right)_{j \in J}$, where $T_{j}$ is a continuous Archimedean $t$-norm with additive generator $t_{j}$ for all $j \in J$. Given a mapping $I:[0,1]^{2} \rightarrow[0,1]$, the following items are equivalent:

i) I is a fuzzy implication function invariant with respect to T-powers, satisfying $(\mathbf{I P})$ and $I\left(b_{j}, y\right)=0$ for all $a_{j} \leq y<b_{j}$ and for all $j \in J$ with $b_{j} \neq 1$.

ii) There exist increasing mappings $\varphi_{j}:[0,1] \rightarrow[0,1]$ with $\varphi_{j}(0)=0$ such that $I$ is given by

$$
I(x, y)= \begin{cases}1 & \text { if } x \leq y, \\ \varphi_{j}\left(\frac{t_{j}\left(\frac{x-a_{j}}{b_{j}-a_{j}}\right)}{t_{j}\left(\frac{y-a_{j}}{b_{j}-a_{j}}\right)}\right) & \text { if } x, y \in\left[a_{j}, b_{j}\right] \text { and } b_{j}>x>y>a_{j} \text { or }(x, y)=\left(b_{j}, a_{j}\right), \\ 0 & \text { if } x>a_{j}>y \text { for some } j \in J,\end{cases}
$$

and the remaining values $I\left(x, a_{j}\right)$ and $I\left(b_{j}, y\right)$ preserve the monotonicity conditions.

PROOF. Let us suppose first that $I$ is a fuzzy implication function invariant with respect to $T$-powers, satisfying (IP) and $I\left(b_{j}, y\right)=0$ for all $a_{j} \leq y<b_{j}$ and for all $j \in J$ with $b_{j} \neq 1$. Given any fixed $j \in J$, note that for all $\left.x \in\right] a_{j}, b_{j}[$ we also have that $\left.x_{T}^{(r)} \in\right] a_{j}, b_{j}$ [ for all real numbers $r>0$ and, in fact, we can write:

$$
\left.x_{T}^{(r)}=a_{j}+\left(b_{j}-a_{j}\right)\left(\frac{x-a_{j}}{b_{j}-a_{j}}\right)_{T_{j}}^{(r)} \quad \text { for all } \quad x, y \in\right] a_{j}, b_{j}[,
$$

or equivalently

$$
\left.x_{T_{j}}^{(r)}=\frac{\left(a_{j}+\left(b_{j}-a_{j}\right) x\right)_{T}^{(r)}-a_{j}}{b_{j}-a_{j}} \quad \text { for all } \quad x, y \in\right] 0,1[.
$$

Let us consider the function $I_{j}:[0,1]^{2} \rightarrow[0,1]$ given by

$$
I_{j}(x, y)=I\left(a_{j}+\left(b_{j}-a_{j}\right) x, a_{j}+\left(b_{j}-a_{j}\right) y\right) \quad \text { for all } \quad x, y \in[0,1] .
$$


Taking into account the equality given in (8), for all $x, y \in] 0,1[$ we obtain

$$
\begin{aligned}
I_{j}\left(x_{T_{j}}^{(r)}, y_{T_{j}}^{(r)}\right) & =I\left(a_{j}+\left(b_{j}-a_{j}\right) x_{T_{j}}^{(r)}, a_{j}+\left(b_{j}-a_{j}\right) y_{T_{j}}^{(r)}\right) \\
& =I\left(\left(a_{j}+\left(b_{j}-a_{j}\right) x\right)_{T}^{(r)},\left(a_{j}+\left(b_{j}-a_{j}\right) y\right)_{T}^{(r)}\right) \\
& =I\left(a_{j}+\left(b_{j}-a_{j}\right) x, a_{j}+\left(b_{j}-a_{j}\right) y\right)=I_{j}(x, y)
\end{aligned}
$$

That is, $I_{j}$ is $T_{j}$-power invariant and clearly satisfies $(\mathbf{I P})$ and consequently by Theorems 10 and 12 , there exists an increasing mapping $\varphi_{j}:[0,1] \rightarrow[0,1]$ with $\varphi_{j}(0)=0$ such that

$$
I_{j}(x, y)= \begin{cases}1 & \text { if } x \leq y \\ \varphi_{j}\left(\frac{t_{j}(x)}{t_{j}(y)}\right) & \text { if } 1>x>y>0 \text { or }(x, y)=(1,0) .\end{cases}
$$

Now, the rest of the proof follows easily.

The structure of the $T$-power based implications in the cases when the continuous t-norm is an ordinal sum can be viewed in Figure 2.

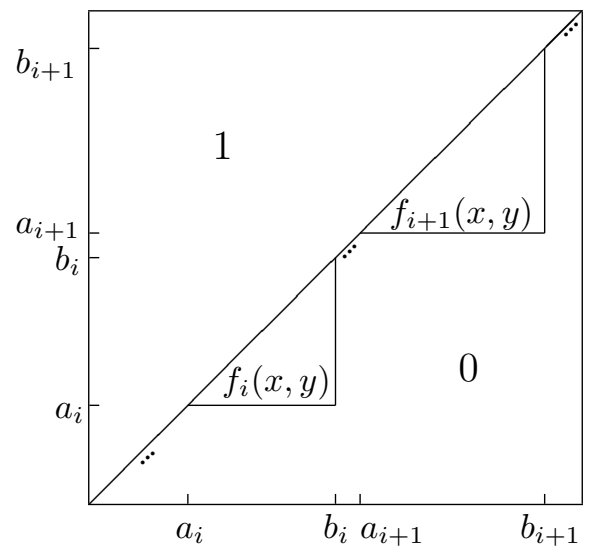

Figure 2: Structure of the fuzzy implication functions satisfying the invariance with respect to $T$-powers when $T$ is the continuous ordinal sum t-norm given by $T=\left(\left\langle a_{j}, b_{j}, T_{j}\right\rangle\right)_{j \in J}$, where $T_{j}$ is an Archimedean t-norm with additive generator $t_{j}$ for all $j \in J$. Moreover, $\varphi_{j}:[0,1] \rightarrow$ $[0,1]$ is an increasing mapping with $\varphi_{j}(0)=0$ and $f_{j}(x, y)$ stands for $f_{j}(x, y)=\varphi_{j}\left(\frac{t_{j}\left(\frac{x-a_{j}}{b_{j}-a_{j}}\right)}{t_{j}\left(\frac{x-a_{j}}{b_{j}-a_{j}}\right)}\right)$ for all $j \in J$.

Remark 4. Note that the above theorem includes all possible cases of continuous t-norms and, in particular, Theorems 10 and 12 become particular cases of it. Specifically, we have

1. When $T$ is the minimum t-norm, all elements are idempotent and there are no Archimedean summands. Consequently, $T$-power invariance gives no condition (see Remark 3), whereas the second case in Equation (7) never applies leading then to the Rescher implication.

2. When $T$ is an Archimedean t-norm there is only one Archimedean summand, i.e., $I$ is a singleton $J=\{1\}$ and $\left[a_{1}, b_{1}\right]=[0,1]$. Consequently, the third condition in item $\left.i\right)$ never applies and the same occurs with the third case in Equation (7). Thus, Theorem 13 reduces to Theorems 10 and 12 in this case.

\subsection{Characterization of $T$-power based implications}

Let us point out that until now the previous results provide in fact a characterization of the class of fuzzy implication functions that satisfy $(\mathbf{I P})$ and the $T$-power invariance property. As we have already commented, and based on the results before, this class contains the class of all $T$-power based implication functions, but also many other 
implications (see Examples 2 and 3). Thus, to characterize the class of $T$-power based implication functions itself, we need to consider some additional condition.

This will be our goal for the rest of the section. Note that $T$-power based implication functions are such that all their vertical sections $I^{T}(x,-)$ with $\left.x \in\right] 0,1[$ are right-continuous at zero when $T$ is Archimedean, and when $T$ is an ordinal sum of the form $T=\left(\left\langle a_{j}, b_{j}, T_{j}\right\rangle\right)_{j \in J}$ then the vertical sections $I^{T}(x,-)$ with $\left.x \in\right] 0,1[$ are right-continuous at all points $\left\{a_{j}, j \in J\right\}$. Moreover, let us prove that they also satisfy the following result.

Proposition 14. Let $T$ be a continuous $t$-norm and $I^{T}$ the corresponding $T$-power based implication. Then

$$
I^{T}(x, y) I^{T}(y, z)=I^{T}(x, z) \text { for all } x>y>z .
$$

Proof. The result is trivial for $T=\min$. If $T$ is Archimedean, for all $x>y>z$ we have

$$
I^{T}(x, y) I^{T}(y, z)=\frac{t(x)}{t(y)} \frac{t(y)}{t(z)}=\frac{t(x)}{t(z)}=I^{T}(x, z) .
$$

Finally, if $T$ is an ordinal sum a similar reasoning proves the result.

Now, we will denote by $\mathcal{C} \mathcal{T}_{\mathcal{P} B \mathcal{I}}$ the class of all power based fuzzy implication functions with respect to a continuous t-norm, and by $\mathcal{A} \mathcal{T}_{\mathcal{P} \mathcal{B} I}$ the subclass of all power based fuzzy implication functions with respect to a continuous Archimedean t-norm. That is:

$$
\mathcal{C} \mathcal{T}_{\mathcal{P B I}}=\left\{I^{T} \mid T \text { is a continuous t-norm }\right\}, \quad \mathcal{A} \mathcal{T}_{\mathcal{P B I}}=\left\{I^{T} \mid T \text { is a continuous Archimedean t-norm }\right\} .
$$

Note that the Rescher implication is in $\mathcal{C} \mathcal{T}_{\mathcal{P B I}}$ since it is the min-power based implication, but it is not in $\mathcal{A} \mathcal{T}_{\mathcal{P B I}}$. For this reason we will also use the notation $\mathcal{A T}_{\mathcal{P B I}}{ }^{+}=\mathcal{A} \mathcal{T}_{\mathcal{P B I}} \cup\left\{I_{\mathbf{R S}}\right\}$.

With this notation we can give the characterization of both classes of fuzzy implication functions as follows:

Theorem 15. Let $I:[0,1]^{2} \rightarrow[0,1]$ be a mapping. The following items hold:

1. $I \in \mathcal{A T}_{\mathcal{P B I}}{ }^{+}$if and only if there exists a continuous Archimedean $t$-norm $T$ such that I is $T$-power invariant, satisfies $(\mathbf{I P}), I(x, y) I(y, z)=I(x, z)$ for all $x>y>z$, and the vertical sections $I(x,-)$ with $x \in] 0,1[$ are right-continuous at zero.

2. $I \in \mathcal{C} \mathcal{T}_{\mathcal{P B I}}$ if and only if there exists a continuous t-norm $T$ such that $I$ is $T$-power invariant, satisfies (IP), $I(x, y) I(y, z)=I(x, z)$ for all $x>y>z$, and the vertical sections $I(x,-)$ with $x \in] a_{j}, b_{j}[$ are rightcontinuous at all points $a_{j}$, where $] a_{j}, b_{j}[$ are the ordinal summands of the continuous $t$-norm $T$.

PROOF. Let us prove both items separately.

1. On the one hand, if $I \in \mathcal{A} \mathcal{T}_{\mathcal{P B I}}{ }^{+}$we have $I=I^{\text {min }}$ or $I=I^{T}$ for some continuous Archimedean t-norm $T$ and we already know that in these cases $I$ is $T$-power invariant, satisfies $(\mathbf{I P}), I^{T}(x, y) I^{T}(y, z)=I^{T}(x, z)$ for all $x>y>z$ and the vertical sections $I(x,-)$ with $x \in] 0,1[$ are right-continuous at zero.

Conversely, if there exists a continuous Archimedean t-norm $T$ with additive generator $t$ such that $I$ is $T$-power invariant and satisfies (IP) then, by Theorems 10 and 12, there exists an increasing mapping $\varphi:[0,1] \rightarrow[0,1]$ with $\varphi(0)=0$ such that $I$ is given by Equation (6). Note that the value $\varphi(1)$ is not relevant. Moreover, since $I$ satisfies $I(x, y) I(y, z)=I(x, z)$ for all $x>y>z$, it must be

$$
\varphi\left(\frac{t(x)}{t(y)}\right) \varphi\left(\frac{t(y)}{t(z)}\right)=\varphi\left(\frac{t(x)}{t(z)}\right) .
$$

Changing $\frac{t(x)}{t(y)}=a$ and $\frac{t(y)}{t(z)}=b$ we obtain that $\varphi$ must satisfy the Cauchy functional equation:

$$
\varphi(a b)=\varphi(a) \varphi(b) \text { for all } a, b \in[0,1] .
$$

Since $\varphi$ is increasing with $\varphi(0)=0$, the only solutions of this equation are the 0 -constant function, the function $\varphi(a)=0$ when $a<1$ and $\varphi(1)=1$, and the function $\varphi(a)=a^{c}$ for some fixed real number $c>0$ (see Section 
13.1 in [16]). The first two possibilities lead to the Rescher implication that is $I \in \mathcal{A} \mathcal{T}_{\mathcal{P B I}}{ }^{+}$, whereas the last one leads to the function

$$
I(x, y)= \begin{cases}1 & \text { if } x \leq y \\ \frac{t(x)^{c}}{t(y)^{c}} & \text { if } 1>x>y>0 \text { or }(x, y)=(1,0)\end{cases}
$$

Let us now deal with the remaining values $I(x, 0)$ and $I(1, y)$. On one hand, it must be satisfied $I(1, y) I(y, z)=$ $I(1, z)$ for all $1>y>z>0$ and consequently

$$
\left.I(1, y)(t(y))^{c}=I(1, z)(t(z))^{c} \quad \text { for all } y, z \in\right] 0,1[.
$$

That is $I(1, y)(t(y))^{c}$ must be constant for all $\left.y \in\right] 0,1[$ and so

$$
\left.I(1, y)=\frac{k}{(t(y))^{c}} \quad \text { for all } y \in\right] 0,1[.
$$

However, since $t(y)$ approaches to 0 when $y$ approaches to 1 and $I(1, y) \in[0,1]$ it must be $k=0$ and consequently $I(1, y)=0$ for all $y \in] 0,1[$. This proves that Equation (9) holds for all points $(1, y)$.

On the other hand, since the vertical sections $I(x,-)$ are right-continuous at 0 for all $x \in] 0,1$ [ we have

$$
I(x, 0)=\lim _{y \rightarrow 0} I(x, y)=\lim _{y \rightarrow 0} \frac{(t(x))^{c}}{(t(y))^{c}}
$$

which ensures that Equation (9) also holds for all points $(x, 0)$.

Finally, taking the generator $t^{\prime}(x)=(t(x))^{c}$ we have proved that $I$ coincides with the $T^{\prime}$-power based implication where $T^{\prime}$ is the Archimedean t-norm generated by $t^{\prime}$, that is, $I$ is again in $\mathcal{A} \mathcal{T}_{\mathcal{P B I}}{ }^{+}$.

2. This case follows in a similar way by applying Theorem 13.

Let us prove finally by given some examples that additional conditions to the $T$-power invariance and (IP) required in the previous theorem are necessary to characterize $T$-power based implications $I^{T}$. We restrict the examples to the case of Archimedean t-norms but they can be easily extended to ordinal sums.

Example 4. Let us give some examples of fuzzy implication functions which are not $T$-power based implications by excluding one by one any of the additional properties in Theorem 15.

i) Note that fuzzy implication functions $I$ given in Example 3-i) satisfy all conditions in Theorem 15-i) except the right-continuity of vertical sections $I(x, 0)$ at point 0 .

ii) On the other hand, the fuzzy implication function $I$ given in Example 3-ii) satisfies all conditions in Theorem 15-i) except the condition $I(x, y) I(y, z)=I(x, z)$ for all $x>y>z$.

\section{An alternative characterization of $T$-power based implications}

In the previous section, we have presented a characterization of the family of $T$-power based implications relying on the invariance property with respect to powers of t-norms. However, that characterization does not provide the concrete t-norm $T$ from which the $T$-power based implication is generated. Therefore, in this section, we want to give an alternative characterization of $T$-power based implications which, in addition to characterize the family, gives the concrete t-norms from which they are generated. This characterization does not make explicitly use of the power invariant property. On the contrary, it will be mainly based on the property given in Proposition 14.

Let us begin with the characterization of $T$-power based implications generated from a nilpotent Archimedean t-norm.

Proposition 16. Let $I:[0,1]^{2} \rightarrow[0,1]$ be a binary function. Then $I$ is a $T$-power based implication for some nilpotent Archimedean t-norm $T$ if and only if the following properties hold: 
a) I satisfies $(\mathbf{O P})$,

b) $I(x, y) \cdot I(y, 0)=I(x, 0)$ for all $x>y$,

c) the horizontal section $I(-, 0):[0,1] \rightarrow[0,1]$ is a strictly decreasing and continuous function with $I(1,0)=0$.

Moreover, in this case the $t$-norm $T$ is the nilpotent Archimedean t-norm with additive generator $t(x)=I(x, 0)$ for all $x \in[0,1]$.

Proof. Let us prove first that if $I$ is a $T$-power based implication with $T$ a nilpotent Archimedean t-norm, then $I$ satisfies these three properties. Indeed, $I$ satisfies (OP) and Property c) by Propositions 8 and 9-(ii) in [23], respectively. Moreover, setting $z=0$ in Proposition 14, we obtain that the following property holds:

$$
I(x, y) \cdot I(y, 0)=I(x, 0), \text { for all } x>y>0 .
$$

This property also holds for $y=0$ since $I(x, 0) \cdot I(0,0)=I(x, 0)$ by $(\mathbf{O P})$ and therefore, Property b) is satisfied. Finally, since additive generators of Archimedean t-norms are unique up to a multiplicative constant, using again Proposition 9-(ii) in [23], we obtain $t(x)=N_{I}(x)=I(x, 0)$ for all $x \in[0,1]$.

Reciprocally, first, by $(\mathbf{O P})$, we get that $I(x, y)=1$ for all $x \leq y$. Let us define $t(x)=I(x, 0)$. First, due to Property c), $t$ is continuous and strictly decreasing with $t(1)=I(1,0)=0$ and $t(0)=I(0,0)=1$ by (OP). Thus, it is an additive generator of some nilpotent Archimedean t-norm. Using this generator and Property $b)$, we get that for all $x>y$,

$$
I(x, y)=\frac{I(x, 0)}{I(y, 0)}=\frac{t(x)}{t(y)} .
$$

Note that for all $x>y$, it holds that $I(y, 0)>0$ since $N_{I}(x)=I(x, 0)$ is strictly decreasing and consequently, $I(y, 0)>I(x, 0) \geq I(1,0)=0$. To sum up, we have proved that

$$
I(x, y)= \begin{cases}1 & \text { if } x \leq y \\ \frac{t(x)}{t(y)} & \text { if } x>y\end{cases}
$$

That is, $I=I^{T}$ for some nilpotent Archimedean t-norm $T$.

The characterization of $T$-power based implications generated from strict Archimedean t-norms is more complex.

Proposition 17. Let $I:[0,1]^{2} \rightarrow[0,1]$ be a binary function. Then $I$ is a T-power based implication for some strict Archimedean t-norm $T$ if and only if I satisfies $(\mathbf{O P})$ and there exists $k \in(0,1]$ such that the following properties hold:

a) $I(x, y) \cdot I(y, z)=I(x, z)$ for all $x>y>z>0$ and $z<k$,

b) $\varphi_{z}:(z, 1] \rightarrow[0,1]$ defined by $\varphi_{z}(x)=I(x, z)$ is a strictly decreasing and continuous function for all $0<z<$ $k$,

c) $I(x, 0)=0$ for all $x>0$,

d) $I(1, y)=0$ for all $y<1$,

e) $I(x, 0)=\lim _{y \rightarrow 0} I(x, y)$.

Proof. Let us prove first that if $I$ is a $T$-power based implication with $T$ a strict Archimedean t-norm, then $I$ satisfies all the aforementioned properties. Indeed, $I$ satisfies $(\mathbf{O P})$ by Proposition 8 in [23] and Property a) by Proposition 14. Moreover, Property b) is satisfied since from the structure of $T$-power based implications with strict Archimedean t-norms given in Proposition 3, we retrieve that

$$
\varphi_{z}(x)=I(x, z)=\frac{t(x)}{t(z)}
$$


which is a strictly decreasing and continuous function for all $0<z<k$. Finally, Properties c) to e) are also proved trivially by using Proposition 3 .

Reciprocally, for all $0<z<k$ and $x>y>z$, by Properties a) and b), it holds that

$$
I(x, y)=\frac{I(x, z)}{I(y, z)}=\frac{\varphi_{z}(x)}{\varphi_{z}(y)} .
$$

Note that $I(y, z) \neq 0$ since $I(x, z)<I(y, z)$ whenever $x>y$ due to the strictly decreasingness of $I(\cdot, z)$. Now, let us define for all $0<z<k$, functions $t_{z}:(z, 1] \rightarrow[0,+\infty)$ given by

$$
t_{z}(x)=\frac{\varphi_{z}(x)}{\varphi_{z}(k)} .
$$

It holds trivially that $t_{z}(1)=0$ by Property d). Moreover, by construction, $t_{z}(k)=1$ and

$$
I(x, y)=\frac{t_{z}(x)}{t_{z}(y)}
$$

for all $0<z<k$ and $x>y>z$. Consider now this family $\left(t_{z}\right)_{0<z<k}$. Let us prove that for $z \leq z^{\prime}, t_{z}$ is an extension of $t_{z^{\prime}}$, that is, $t_{z}(x)=t_{z^{\prime}}(x)$ for all $x>z^{\prime}$. Using Property a), we obtain

$$
t_{z}(x)=\frac{I(x, z)}{I(k, z)}=\frac{I\left(x, z^{\prime}\right) \cdot I\left(z^{\prime}, z\right)}{I\left(k, z^{\prime}\right) \cdot I\left(z^{\prime}, z\right)}=\frac{I\left(x, z^{\prime}\right)}{I\left(k, z^{\prime}\right)}=t_{z^{\prime}}(x) .
$$

Let us define $t:(0,1] \rightarrow[0, \infty)$ as $t(x)=t_{z}(x)$ for $z<k$ and $z<x$. It is easy to check that $t$ is a strictly decreasing and continuous function such that $t(1)=0$ and

$$
I(x, y)=\frac{t(x)}{t(y)}
$$

for all $x>y>0$. Define $t(0)=\lim _{y \rightarrow 0} t(y)$. For all $x>0$, we have by Properties c) and e) that

$$
I(x, 0)=\lim _{y \rightarrow 0} I(x, y)=\lim _{y \rightarrow 0} \frac{t(x)}{t(y)}=\frac{t(x)}{t(0)}=0 .
$$

Since $t(x)>0$ for $x<1$, it must be $t(0)=\infty$. Consequently, $t$ is the additive generator of a strict $\mathrm{t}$-norm $T$ and $I=I^{T}$.

Finally, we give an analogous characterization in the general continuous case, that is, when $T$ is an ordinal sum of the form $T=\left(\left\langle a_{j}, b_{j}, T_{j}\right\rangle\right)_{i \in J}$, where $T_{j}$ are continuous Archimedean t-norm with additive generators $t_{j}$ for all $j \in J$.

Proposition 18. Let $I:[0,1]^{2} \rightarrow[0,1]$ be a binary function. Then $I$ is a T-power based implication for some ordinal sum $t$-norm $T=\left(\left\langle a_{i}, b_{i}, T_{i}\right\rangle\right)_{i \in J}$ if and only if I satisfies $(\mathbf{O P})$ and for all $j \in J$, one of the following cases hold:

i) If $T_{j}$ is non-strict, then the following properties hold:

a) if $x, y \in\left[a_{j}, b_{j}\right]$ with $x>y$, then $I(x, y) \cdot I\left(y, a_{j}\right)=I\left(x, a_{j}\right)$,

b) the summand horizontal section $I\left(-, a_{j}\right):\left[a_{j}, b_{j}\right] \rightarrow[0,1]$ is a strictly decreasing and continuous function with $I\left(b_{j}, a_{j}\right)=0$.

ii) If $T_{j}$ is strict, then there exists $k_{j} \in\left(a_{j}, b_{j}\right]$ such that the following properties hold:

a) $I(x, y) \cdot I(y, z)=I(x, z)$ for all $b_{j} \geq x>y>z>a_{j}$ and $z<k_{j}$.

b) $\varphi_{z}:\left(z, b_{j}\right] \rightarrow[0,1]$ defined by $\varphi_{z}(x)=I(x, z)$ is a strictly decreasing and continuous function for $a_{j}<z<k_{j}$,

c) $I\left(x, a_{j}\right)=0$ for all $x \in\left(a_{j}, b_{j}\right]$,

d) $I\left(b_{j}, y\right)=0$ for all $y \in\left[a_{j}, b_{j}\right)$,

e) $I\left(x, a_{j}\right)=\lim _{y \rightarrow a_{j}} I(x, y)$ for all $x \in\left(a_{j}, b_{j}\right]$.

PROOF. The result follows by adapting adequately to the corresponding summand of the ordinal sum t-norm the proofs of Propositions 16 and 17. 


\section{Conclusions and future work}

The invariance of the truth value of a fuzzy conditional when both the antecedent and the consequent are modified using the same quantifier is the most important property satisfied by the $T$-power based implications. In this paper, we have fully characterized this recently introduced family of fuzzy implication functions using this invariance property. This property is so strong that even those binary functions, not necessarily fuzzy implication functions, that satisfy this property can be characterized (see Theorem 7). Moreover, just by adding (IP) it characterizes the family of $T$-power based implications up to compositions with increasing mappings $\varphi:[0,1] \rightarrow[0,1]$ with $\varphi(0)=0$. To achieve the characterizations of the proper $T$-power based implications, the property

$$
I^{T}(x, y) \cdot I^{T}(y, z)=I^{T}(x, z) \text { for all } x>y>z
$$

introduced in Proposition 14, has proved to be essential, even to achieve in Section 4 some alternative characterizations which do not involve the power invariance property. This property is important because it ensures that $T$-power based implications are unidimensional $T^{\prime}$-preorders with $T^{\prime}$ the product t-norm [8].

As a future work, we want to improve the characterizations presented in this paper. In this direction, we want to reduce the number of required properties and to use more common properties of fuzzy implication functions. Moreover, there are still some open problems regarding $T$-power based implications. In particular, we want to study the properties of the Modus Ponens and the Modus Tollens and to generalize the family to left-continuous t-norms, whose powers can be easily adapted.

\section{Acknowledgements}

This paper has been supported by the Spanish project TIN2016-75404-P AEI/FEDER, UE.

\section{References}

[1] I. Aguiló, J. Suñer, J. Torrens, A characterization of residual implications derived from left-continuous uninorms, Information Science, 180(20), 3992-4005 (2010).

[2] I. Aguiló, J. Suñer, J. Torrens, New types of contrapositivisation of fuzzy implications with respect to fuzzy negations, Information Science, 322, 223-226 (2015).

[3] M. Baczyński, G. Beliakov, H. Bustince Sola, A Pradera (Eds.), Advances in Fuzzy Implication Functions, Studies in Fuzziness and Soft Computing, vol. 300, Springer, Berlin Heidelberg, 2013.

[4] M. Baczyński, P. Grzegorzewski, R. Mesiar, P. Helbina, W. Niemyska, Fuzzy implications based on semicopulas, Fuzzy Sets and Systems,

[5] M. Baczyński, B. Jayaram, Fuzzy Implications, Studies in Fuzziness and Soft Computing, vol. 231, Springer, Berlin Heidelberg, 2008.

[6] M. Baczyński, B. Jayaram, S. Massanet, J. Torrens, Fuzzy Implications: Past, Present, and Future, in J. Kacprzyk and W. Pedrycz (eds.), Springer Handbook of Computational Intelligence, Springer Berlin Heidelberg, pp. 183-202 (2015).

[7] D. Boixader, J. Recasens, Powers with Respect to t-Norms and t-Conorms and Aggregation Functions, in T. Calvo and J. Torrens (eds.), Fuzzy Logic and Information Fusion - To Commemorate the 70th Birthday of Professor Gaspar Mayor, Studies in Fuzziness and Soft Computing, vol. 339, pp. 41-47, Springer, 2016.

[8] D. Boixader, J. Recasens, Generation and Characterization of Fuzzy T-preorders, Applied Artificial Intelligence, 29(5), 514-530 (2015).

[9] J. C. Fodor and M. Roubens. Fuzzy Preference Modelling and Multicriteria Decision Support. Kluwer Academic Publishers, Dordrecht, 1994.

[10] P. Grzegorzewski, Probabilistic implications, Fuzzy Sets and Systems, 226, 53-66 (2013).

[11] P. Grzegorzewski, Survival Implications, in S. Greco et al., Advances in Computational Intelligence: 14th International Conference on Information Processing and Management of Uncertainty in Knowledge-Based Systems, IPMU 2012, Proceedings, Part II, Springer Berlin Heidelberg, 335-344 (2012).

[12] D. Hliněná, M. Kalina, P. Král, Implication Functions Generated Using Functions of One Variable, in M. Baczyński et al. (eds.), Advances in Fuzzy Implication Functions, Studies in Fuzziness and Soft Computing, vol. 300, pp. 125-153, Springer-Verlag, 2013.

[13] E.E. Kerre, M. De Cock, Linguistic Modifiers: An Overview, in G. Chen, M. Ying and K.-Y. Cai (eds.), Fuzzy Logic and Soft Computing, pp. 69-85 (1999).

[14] E.P. Klement, R. Mesiar, E. Pap, Triangular norms, Kluwer Academic Publishers, Dordrecht, 2000.

[15] A. Kolesárová, R. Mesiar, T. Rückschlossová, Power stable aggregation functions, Fuzzy Sets and Systems, 240, 39-50 (2014).

[16] M. Kuczma, An Introduction to the Theory of Functional Equations and Inequalities, Ed. A. Gilányi. 2nd ed. Birkhäuser Basel, 2009.

[17] M. Mas, M. Monserrat, J. Torrens, E. Trillas, A survey on fuzzy implication functions, IEEE Transactions on Fuzzy Systems, 15(6), 1107-1121 (2007).

[18] S. Massanet, J. Torrens, On a new class of fuzzy implications: h-Implications and generalizations, Information Science, 181, 2111-2127 (2011).

[19] S. Massanet, J. Torrens, On the characterization of Yager's implications, Information Science, 201, 1-18 (2012). 
[20] S. Massanet, J. Torrens, Threshold generation method of construction of a new implication from two given ones, Fuzzy Sets and Systems, 205, 50-75 (2012).

[21] S. Massanet, J. Torrens, On the vertical threshold generation method of fuzzy implication and its properties, Fuzzy Sets and Systems, 226, 232-252 (2013).

[22] S. Massanet, J. Torrens, Characterization of fuzzy implication functions with a continuous natural negation satisfying the law of importation with a fixed t-norm, IEEE Transactions on Fuzzy Systems, 25(1), 100-113 (2017).

[23] S. Massanet, J. Recasens, J. Torrens, Fuzzy implication functions based on powers of continuous t-norms, International Journal Approximate Reasoning, 83, 265-279 (2017).

[24] M. Mizumoto, H.-J. Zimmermann, Comparison of fuzzy reasoning methods, Fuzzy Sets and Systems, 8, 253-283 (1982).

[25] A. Pradera, G. Beliakov, H. Bustince, B. De Baets, A review of the relationships between implication, negation and aggregation functions from the point of view of material implication, Information Science, 329, 357-380 (2016).

[26] N. Rescher, Many-valued logic. McGraw-Hill, New York, 1969.

[27] Y. Su, A. Xie, H. Liu, On ordinal sum implications, Information Sciences, 293, 251-262 (2015).

[28] E. Trillas, M. Mas, M. Monserrat, J. Torrens, On the representation of fuzzy rules, International Journal of Approximate Reasoning, 48, 583-597 (2008).

[29] N.R. Vemuri, B. Jayaram, Fuzzy implications: Novel generation process and the consequent algebras, Commun. Comput. Inf. Sci. Ser., 298, 365-374 (2012).

[30] N.R. Vemuri, B. Jayaram, The $\circledast$-composition of fuzzy implications: Closures with respect to properties, powers and families, Fuzzy Sets and Systems, 275, 58-87 (2015).

[31] C. L. Walker, E. A. Walker, Powers of t-norms, Fuzzy Sets and Systems, 129, 1-18 (2002).

[32] R. R. Yager, On some new classes of implication operators and their role in approximate reasoning, Information Science, 167, 193-216 (2004).

[33] L.A. Zadeh. Similarity relations and fuzzy orderings. Information Sciences 3, 177-200 (1971). 